\title{
A 3D CZT prototype for Laue lens telescope
}

\author{
Ezio Caroli ${ }^{1}$, Natalia Auricchio, \\ INAF/IASF-Bologna \\ Via Gobetti 101, I-40129 Bologna, Italy \\ E-mail:caroli@iasfbo.inaf.it; auricchio@iasfbo.inaf.it
}

\section{Carl Budtz-Jorgensen, Irfan Kuvvetli}

NSI/DTU, Technical University of Denmark

Juliane Maries vej 30, DK-2100 Copenhagen, Denmark

E-mail: carl@space.dtu.dk,irfan@space.dtu.dk

\section{Stefano del Sordo}

INAF/IASF-Palermo,

Via Ugo La Malfa 153, I-90146 Palermo, Italy

E-mail: Delsordo@ifc.inaf.it

\section{Rui M. Curado da Silva}

Departamento de Fisica, Universidade de Coimbra,

P-3004-516 Coimbra, Portugal

E-mail: ruisilva@saturno.fis.uc.pt

\section{Luciano Milano}

Dipartimento di Fisica, Università di Ferrara,

Via Saragat 1, I-44100 Ferrara, Italy

E-mail:milano@fe.infn.it

\section{Mauro E. Quadrini, Fabio Casini}

INAF/IASF-Milano,

Via Bassini 15, I-20133 Milano, Italy

E-mail:mauro@iasf-milano.inaf.it;

\section{Andrea Zappettini}

CNR/IMEM,

Parco Area delle Scienze 37/A, I- 43124 Parma, Italy

E-mail: zapp@imem.cnr.it

\section{Massimiliano Zanichelli}

Dipartimento di Fisica, Università di Parma,

Parco Area delle Scienze 37/A, I- 43124 Parma, Italy

E-mail: massimiliano.zanichelli@fis.unipr.it

\footnotetext{
$1 \quad$ Speaker
} 


\title{
Lorenzo Natalucci, Pietro Ubertini
}

INAF/IASF-Roma,

Via Fosso del Cavaliere, I-00133 Frascati, Italy

E-mail: natalucci@iasf-roma.inaf.it, ubertini@iasf-roma.inaf.it

\begin{abstract}
The importance of hard X-ray astronomy $(>10 \mathrm{keV})$ is now widely recognized. Recently both ESA (Cosmic Vision) and NASA (Decadal Survey) have indicated in their guidelines for $\mathrm{X}$ - and $\gamma$-ray astronomy in the next decade the development of innovative instrumentation operating in the hard $\mathrm{X}$ - and soft gamma ray range where important scientific issues are still open, exploiting high sensitivity for spectroscopic imaging and polarimetric observations. In this framework, the development of new focusing optics based on Laue lenses operating from $\sim 60 \mathrm{keV}$ up to a few $\mathrm{MeV}$ is particularly challenging.

We describe the design concepts and the undergoing development of a three dimensional (3D) position sensitive device suitable for use as the basic unit of a high efficiency focal plane detector for a Laue lens telescope. The sensitive unit is a drift strip detector based on a CZT crystal, $\left(19 \times 8 \mathrm{~mm}^{2}\right.$ area, $2.4 \mathrm{~mm}$ thick), irradiated transversally to the electric field direction. The anode is segmented into 8 detection cells, each comprising one collecting strip and 8 drift strips. The drift strips are biased by a voltage divider, whereas the anode strips are held at $0 \mathrm{~V}$. The cathode is divided in 4 horizontal strips for the reconstruction of the $\mathrm{Z}$ interaction position. The 3D prototype will be made by packing 8 linear modules, each composed of one basic sensitive unit, bonded onto a supporting layer. The readout electronics will implement the new RENA-3 Asic and the data handling system will use custom designed FPGA based electronics to provide both the ASIC operation and the acquisition logic.
\end{abstract}

The Extreme sky: Sampling the Universe above $10 \mathrm{keV}$ - extremesky2009

Otranto (Lecce) Italy

October 13-17, 2009

\section{Introduction}

In high energy astronomy, the next decade will be characterized by the development of new space instrumentation working in the energy range from the $\mathrm{keV}$ to the $\mathrm{MeV}$ region, where many important scientific issues are still open [1]. In particular, the development of new instrumentation based on focusing instruments based on Laue lenses operating from $\sim 50 \mathrm{keV}$ up to a few $\mathrm{MeV}$ is particularly challenging [2].

To exploit the performance of Laue lenses telescope, new focal plane detectors for high energy (50-1000 keV) shall be developed providing: (i) high efficiency $(>80 \%)$ up to the $\mathrm{MeV}$ region; (ii) moderate spatial resolution $(1-3 \mathrm{~mm}$ ); (iii) $3 \mathrm{D}$ sensitivity to photon interaction position for background rejection and high sensitivity polarimetry measurements (minimum detectable polarisation of few $\%$ in $10^{5} \mathrm{~s}$ ); (iv) good spectroscopic resolution (few \% FWHM at $100 \mathrm{keV})$.

Using the up to date room temperature semiconductor technology (CZT), we have proposed a focal plane detector configuration [3] that could reach several $\mathrm{cm}$ thickness without increasing too much the number of layers and pixels (i.e. electronic channels) by coupling two basic ideas for the CZT sensors configuration that are described in the following section. 


\section{The detector sensitive basic unit}

The proposed 3D prototype is based on the use of CZT crystals in the Planar Transverse Field (PTF) configuration, as proposed by our group in the 1990's [4], in which the direction of the impinging photons (i.e. the optical axis of the sensor) is perpendicular to the charge collecting field. This configuration allows to increase the photon absorption thickness without increasing the charge collection distance (i.e. avoiding severe spectroscopic performance degradation) (Fig. 1a).

The drawback of the PTF irradiating geometry is that all the positions between the collecting electrodes are uniformly hit by radiation leading to a stronger effect of the difference in charge collection efficiency in the spectroscopic performance with respect to the standard irradiation configuration (PPF) through the cathode (Fig. 1b).

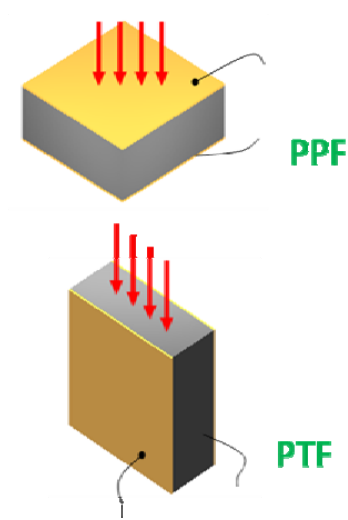

(a)

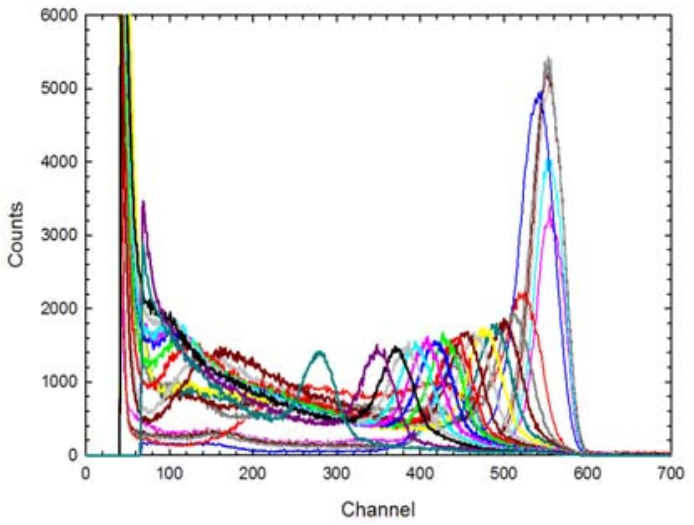

(a)

Figure 1. (a) Irradiating configurations for a solid state detector; (a) The energy spectra (measured with a IMEM/Italy CZT for a $120 \mathrm{keV}$ beam at the ESRF/Grenoble) at different distances from the cathode, showing the effect on the charge collection efficiency and on the energy resolution: at the cathode (highest channel) $\triangle E / E(F W H M$ ) is $\sim 6.9 \%$ while at $0.5 \mathrm{~mm}$ (channel $\sim 300$ ) become $\sim 13.2 \%$.

Therefore in order to recover from this spectroscopic degradation and to improve the CZT sensitive unit performance, we have decided to use an anode made of an array of microstrips in a drift configuration: a thin collecting anode strip surrounded by guard strips with decreasing bias voltage. This anode configuration, proposed and studied at NSI/DTU in Copenhagen [5], allows the detector to be more sensitive to electron collection and less sensitive to hole collection, avoiding the degradation of the spectroscopic response by the charge loss due to the hole trapping and providing a more uniform spectroscopic

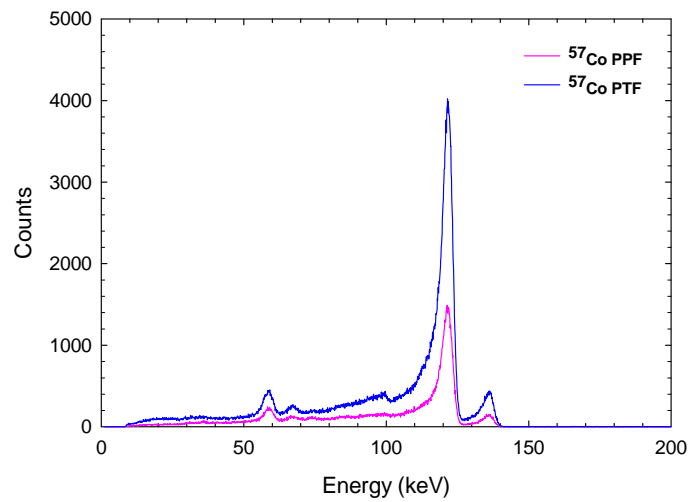

Figure 2. The spectra obtained with a CZT drift strip detector similar to the one proposed in PPF and PTF irradiation configurations. The detector shows a good spectroscopic response in both cases (3\% FWHM at $122 \mathrm{keV})$ while the efficiency is clearly improved for PTF geometry. 
response (i.e. independent from the distance of the interaction from the collecting electrodes as shown in Fig. 2).

Finally in order to obtain 3D sensitivity for the photon interaction position, the cathode is segmented into 4 strips in the direction orthogonal to the anode ones. Therefore the final prototype becomes equivalent to a stack of thinner CZT horizontal layers with the advantage of not having any passive material between each layer.

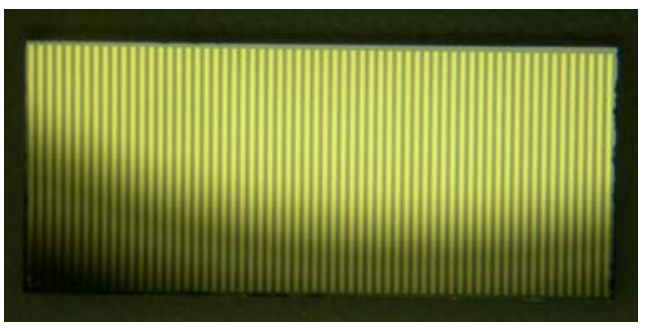

Figure 3. The CZT sensitive unit seen from the anode side that consists of a set of 63 micro-strips. The strips are $0.15 \mathrm{~mm}$ wide and have a vitch of $0.3 \mathrm{~mm}$.

Fig. 3 shows the final CZT sensor unit that was realized using REDLEN Tech. CZT wafers cutting 8 single crystals of $19 \times 8 \times 2.4 \mathrm{~mm}^{3}$. The microstrip set is divided in 8 equivalent pixels with a pitch of $2.4 \mathrm{~mm}$, being only 8 strips connected to the readout electronics, while the others act as drift strips used to shape the charge collecting, through a proper decreasing bias field. Each collecting strip is surrounded by 4 drift strips on each side.

\section{The prototype electronics}

The signals from the 64 anode collecting strips and the 32 cathodes are readout by three RENA-3 ASIC (Fig. 4), placed on the analog front-end electronics (AFEE) [5]. The RENA-3 ASIC is a 36-channel charge sensitive amplifier self-triggering. Each channel includes a lownoise preamplifier, a shaper with sample/hold, and in addition a fast shaper that gives a trigger signal for coincident event detection. The signal range is selectable channel by channel over two full scales (equivalent to $200 \mathrm{keV}$ and $1.3 \mathrm{MeV}$ for $\mathrm{CZT}$ ) as the peaking time that can ranges from 0.1 to $40 \mu \mathrm{s}$. The comparator thresholds can be adjusted through an 8 bit DAC on each channel in order to obtain an accurate and uniform threshold setting. A pole-zero cancellation circuit is available for minimizing pileup errors. All these features are selectable via software. Furthermore, the AFEE implements the detector high voltage power supply, low voltage power supply, the conditioning electronics, the low and high voltage monitors, two temperature and humidity sensors for detector and electronics monitoring

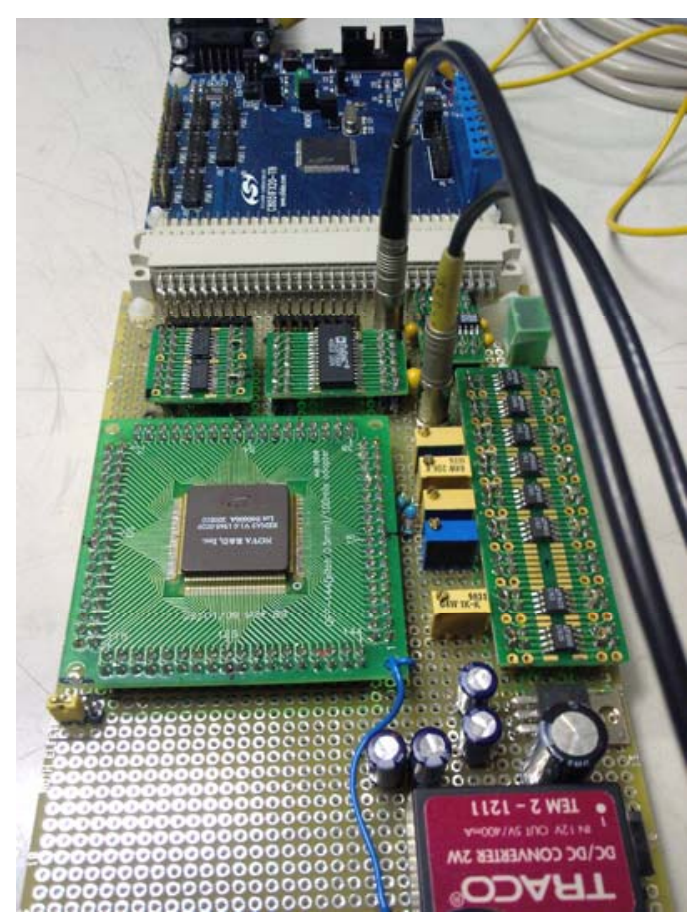

Figure 4. The RENA-3 demo board realized to test the ASIC functionalities before the final AFEE implementation. 


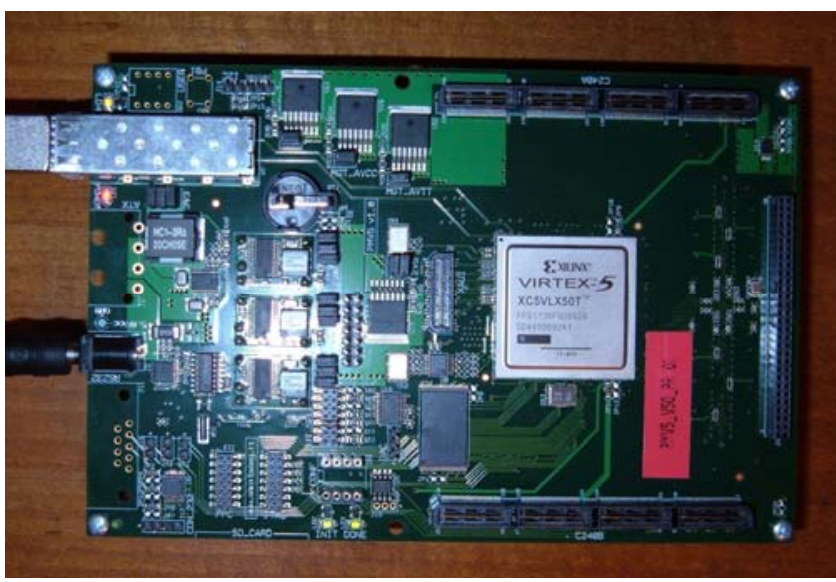

Figure 5. The DHE main board currently under test. The FPGA (a Xlinx VIRTEX 5) core is clearly visible on the right together with the high density connectors to the interface daughter board. of the environmental conditions.

The Data Handling Electronics (DHE) is composed of a daughter board that provides the interface between the AFEE and the main data handling board, shown in Fig. 5. The DHE manages the detector high voltage power supply, the signal amplitude A/D converters, the ASIC parameters configuration, the coincidence logics, the detector operational parameters, and finally prepares the output data stream using a programmable data format (LABVIEW compatible). The main DHE board is based on a FPGA with embedded PPC and will be able to handle up to 128 independent channels (voxels) via an Ethernet link.

\section{The 3D CZT prototype assembly}

After the realisation of the basic sensitive unit, the second step is represented by assembling of the spectrometer linear module (Fig. 6a). We foresee the realisation of 8 linear modules each containing one CZT sensitive unit bonded on ceramic layer $\left(\mathrm{Al}_{2} \mathrm{O}_{3}\right)$. On the ceramic layer each drift strip will be connected to the bias divider, while the anode collecting strips will be connected to front-end electronic channel pads through devoted filter circuits. On the same layer the cathode strip connections will also be realised as the bias circuit and I/O pins to the AFEE electronics.

The third step is the packaging of the 8 linear modules to obtain the 3D position sensitive detector unit with $8 \times 8$ pixels (channels) and a total of $64 \times 4$ voxels. Fig. $6 \mathrm{~b}$ shows the current design of this support, that allows to flow an inert gas (e.g. N) inside the detector box for temperature stabilisation and humidity control (Fig. 6b). This box will be closed on the top by a light tight cover with a Be window. This aluminium detector box will be mechanical connected to a ground referenced metallic frame on the centre of the AFEE board where the height linear modules I/O connectors will be mounted.

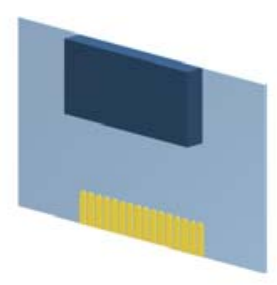

(a)

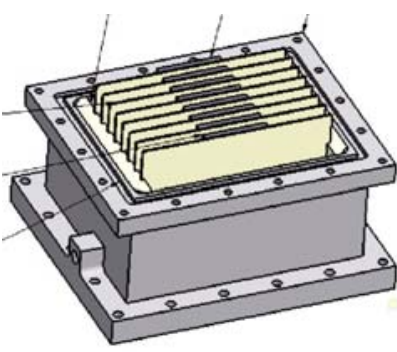

(b)

Figure 6. The 3D CZT detector unit assembling scheme. (a) the CZT linear module; (b) the linear module packaging to form the detector unit. 
Finally all the prototype subsystems describe before will be integrated (see Fig. 7 for a possible layout) to built the $3 \mathrm{D}$ CZT prototype which the main characteristics are summarized in the Table 1.

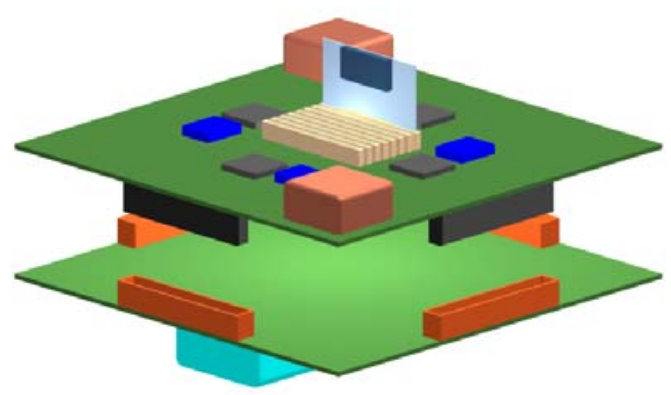

Figure 7. A possible layout to integrate the detector unit with the AFEE (top green layer) and DHE (bottom green layer).
Table I. 3D CZT prototype main characteristics

\begin{tabular}{|c|c|c|}
\hline Item & Characteristics & $\mathrm{N}$. \\
\hline CZT units & $8 \times 19 \times 2.4 \mathrm{~mm}^{3}$, in PTF & 8 \\
\hline Linear modules & $100 \times 30 \mathrm{~mm}^{3}$, Alumina & 8 \\
\hline Active pixels & $8 \times 8\left(2.4 \times 2.4 \mathrm{~mm}^{2}\right)$ & 64 \\
\hline Active voxels. & $8 \times 8 \times 4\left(2.4 \times 2.4 \times 2 \mathrm{~mm}^{3}\right)$ & 256 \\
\hline Surface/Volume & $3.65 \mathrm{~cm}^{2} / 2.92 \mathrm{~cm}^{3}$ & \\
\hline Electronics & $128 \mathrm{channels}^{3}$ & \\
\hline AFEE & Rena-3 ASIC & 3 \\
\hline CZT/ASIC's bias & $300 \mathrm{~V} / 4 \mathrm{~V}$ by DC-DC unit & 2 \\
\hline ADC /DPE & 4 ADC's, 1 FPGA & \\
\hline I/O Interface & LAN & \\
\hline Power & $\sim 20 \mathrm{~W}$ & \\
\hline
\end{tabular}

\section{Conclusions}

Currently we are realising the AFEE board and the definitive DHE board is under test. In the meanwhile we are going to start the realisation of the CZT linear modules. The final 3D CZT detector assembling is scheduled for next May and successively we have foreseen different test campaigns to study the performances using both radioactive sources and beam facilities.

\section{References}

[1] J. Knödlseder; et. al., GRI: the Gamma-ray Imager mission, in "Space Telescopes and Instrumentation II: Ultraviolet to Gamma Ray”, M.J.L. Turner, and G. Hasinger Eds, Proc. of SPIE Vol. 6266, p. 626623, (2006)

[2] F. Frontera, et al., Focusing of gamma-rays with Laue lenses: first results, in "Ultraviolet to Gamma Ray”, edited by M.J.L. Turner, K.A. Flanagan Eds, Proc. of SPIE Vol. 7011, 70111R, (2008)

[3] E. Caroli, et al., A three-dimensional CZT detector as a focal plane prototype for Laue Lens telescope, in "Ultraviolet to Gamma Ray", M.J.L. Turner, K.A. Flanagan Eds., Proc. of SPIE Vol. 7011, p. 70113G, (2008)

[4] F. Casali, et al., IEEE Trans. Nucl. Sci., Vol. 39, p. 598 (1992).

[5] I. Kuvvetli and C. Budtz-Jørgensen, Pixelated CdZnTe drift detectors, IEEE Trans. Nucl. Sci, Vol. 52, N. 5, p. 1975 (2005)

[6] T. O. Tumer, et al, Performance of RENA-3 IC with position-sensitive solid-state detectors, in "Hard X-Ray, Gamma-Ray, and Neutron Detector Physics X", A. Burger; L.A. Franks; R.B. James, Eds, Proc. of SPIE Vol. 7079, p. 7079F (2008). 